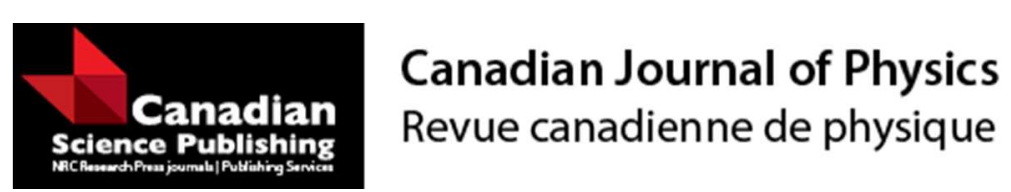

\title{
Approaching special relativity in complex time
}

\begin{tabular}{|r|l|}
\hline Journal: & Canadian Journal of Physics \\
\hline Manuscript ID & cjp-2017-0089 \\
\hline Manuscript Type: & Article \\
\hline Date Submitted by the Author: & 03-Feb-2017 \\
\hline Complete List of Authors: & Spears, James; University of Waterloo, Science \\
\hline Keyword: & $\begin{array}{l}\text { Special Relativity, Logic, set theory, and algebra, Mathematical Physics, } \\
\text { Theoretical Physics, High level categorization }\end{array}$ \\
\hline $\begin{array}{r}\text { Please Select from this Special } \\
\text { Issues list if applicable: }\end{array}$ & N/A \\
\hline & \multicolumn{2}{|l}{} \\
\hline
\end{tabular}

SCHOLARONE ${ }^{\mathrm{m}}$

Manuscripts 


\title{
Approaching special relativity in complex time \\ PACS: $03.30 .+\mathrm{p}, 02.10 .-\mathrm{v}$
}

by: James W. Spears

\author{
Faculty of Science, \\ University of Waterloo \\ 200 University Avenue West \\ Waterloo, Ontario, Canada N2L 3G1, \\ Phone: (519) 888-4567 \\ Fax: (519) 746-8115 \\ email: jspears@uwaterloo.ca
}

April 6, 2017 


\begin{abstract}
Algebraic techniques are employed to extend time in the complex plane. We then use the framework to examine the fundamental postulates of special relativity. Generalizing the notion of time introduces the reader to a unique perspective on the universality of light speed according to Einstein. In the context of complex time we will describe the mathematical conditions in which light speed universality arises. Using high level terminology we then develop a categorization scheme applicable to physical theories which assume light speed is universal.
\end{abstract}

\title{
1 Introduction
}

If interpretation of the founding postulates of a physical theory have influence on the interpretation of experimental results; it is of important consideration to illustrate founding principles in the clearest manner possible. Paying careful attention to definition we will create a vocabulary used to describe properties of time. And in doing so, facilitate an efficient discussion on the foundation of Einstein's theory of special relativity.

Our focus will be restating the postulates of special relativity upon extending time in the complex plane. Any generalizations to the foundations of special relativity will be accompanied by supporting mathematical arguments inspired by the geometry of complex numbers. Using vector notation we will provide more physically meaningful definitions and eliminate much of the abstraction before applying these ideas to time.

When referring to mathematical concepts defined herein, an italicized font will be used to indicate significance beyond the usual interpretation of the word. Except in quotations, where the original author may have used this font for separate reasons. 


\section{Developing complex time in vector notation}

Consider now two orthogonal axes represented in Cartesian coordinates. Let the horizontal axis $t_{p}$ represent the past, and the vertical axis $t_{f}$ the future. Continue by defining the time vector $\vec{t}$ such that

$$
\vec{t}=\left(\begin{array}{c}
t_{p} \\
t_{f}
\end{array}\right)
$$

When these vector components satisfy

$$
t_{p}<t_{f}
$$

we will say the time vector $\vec{t}$ is future oriented. When the vector components of $\vec{t}$ satisfy

$$
t_{p}>t_{f}
$$

we say $\vec{t}$ is past oriented. For vector components of $\vec{t}$ where

$$
t_{p}=t_{f}
$$

we say the vector $\vec{t}$ is present oriented. If we have time elapse into the future it is sensible to reference the angular component $\omega$ of $\vec{t}$ from the real unit vector $\vec{e}_{t}$ where

$$
\vec{e}_{t}=\left(\begin{array}{c}
\frac{-1}{\sqrt{2}} \\
\frac{1}{\sqrt{2}}
\end{array}\right) .
$$

Any point in real time can then be described by scalar multiplication of the unit vector

$$
p \cdot \vec{e}_{t} \mid p \in \mathbb{R},
$$


where we call $p$ the component displacement in the real direction (it should be mentioned for clarity that $\mathbb{R}$ represents the set of real numbers and is not intended to imply real time). Let us now introduce the concept of imaginary time by defining the imaginary unit vector $\vec{e}_{i t}$ such that

$$
\vec{e}_{i t}=\left(\begin{array}{c}
\frac{1}{\sqrt{2}} \\
\frac{1}{\sqrt{2}}
\end{array}\right)
$$

And so any point in imaginary time can be described in a similar fashion

$$
q \cdot \vec{e}_{i t} \mid q \in \mathbb{R}
$$

where $q$ is the component displacement in the imaginary direction. A vector with non-zero component of the imaginary unit vector will by definition have non-zero component displacement which is present oriented. Presently oriented component displacement will serve as our functional definition of imaginary time. We complete the definition of complex time $\zeta$ such that

$$
\zeta:=p \cdot \vec{e}_{t}+q \cdot \vec{e}_{i t} \mid p, q \in \mathbb{R}
$$

where $\zeta$ may then represent any point in complex time by vector addition of the real and imaginary unit vectors and their respective scalar multiples $p$, and $q$. Now that we have a definition of complex time $\zeta$, we may define real and imaginary operators on $\zeta$. We define the real operator

$$
\Re(\zeta):=p
$$

and the imaginary operator

$$
\Im(\zeta):=q
$$


If we choose, we may write the real and imaginary operators in terms of component displacements $p, q$, and temporal angular component $\omega$. These are

$$
\Re(\zeta)=\sqrt{p^{2}+q^{2}} \cdot \cos \omega
$$

and

$$
\Im(\zeta)=\sqrt{p^{2}+q^{2}} \cdot \sin \omega
$$

respectively. Now, we will need to define what the time interval $\Delta t$ shall be represented by. So, let the time interval

$$
\Delta t:=\sqrt{p^{2}+q^{2}}
$$

as the magnitude of $\zeta$. By this definition $\Delta t$ is a strictly positive quantity and that is fine. We have orientation dictated by the temporal angular component $\omega$. For clarity of definition we will continue to use the form

$$
\zeta=\Delta t\left[\cos \omega \cdot \vec{e}_{t}+\sin \omega \cdot \vec{e}_{i t}\right]
$$

where we are explicitly stating the temporal angular component $\omega$.

\section{Degenerate vs. non-degenerate complex time}

As we continue let us refer to $\omega$ as the operational definition of time, or synonymously, the temporal angular component. 


\subsection{Definition: degenerate complex time}

Degenerate complex time is defined $\zeta$, such that $\zeta$ satisfies the equation

$$
\zeta=\Delta t\left[\cos (n \pi) \cdot \vec{e}_{t}+\sin (n \pi) \cdot \vec{e}_{i t}\right] \mid n \in \mathbb{Z}
$$

In cases where the temporal angular component $\omega=n \pi$ for integers $n$, the complex time vector takes the value $\Delta t \cdot \pm \vec{e}_{t}$. In this case, time is said to degenerate such that orientations are either forward or reverse along the real line. For example, the formulation of special relativity which satisfies

$$
\Re(\zeta)= \pm \Delta t
$$

for all possible operational definitions of time can be referred to as: special relativity in degenerate complex time. If all possible operational definitions of time satisfy Eq. (17), we say there exists an absolute operational definition of time.

\subsection{Definition: non-degenerate complex time}

If Eq. (17) is not satisfied for all operational definitions of time, then complex time is defined as being non-degenerate.

\subsection{Definition: relatively degenerate case}

Complex time is defined to be relatively degenerate for two arbitrary operational definitions if

$$
\Delta \omega=n \pi \mid n \in \mathbb{Z}
$$

(All operational definitions in degenerate complex time are also relatively degenerate.) 


\subsection{Definition: relatively non-degenerate case}

Complex time is defined to be relatively non-degenerate for two arbitrary operational definitions if

$$
\Delta \omega \neq n \pi \mid n \in \mathbb{Z} .
$$

(Not all operational definitions in non-degenerate complex time are also relatively non-degenerate.)

\section{Reviewing the fundamental postulates of spe- cial relativity}

The theory of special relativity according to Einstein can be deduced from two fundamental physical postulates.
1) Principle of relativity (Galileo, Newton) [1]: "If a system of coor- dinates $K$ is chosen so that, in relation to it, physical laws hold good in their simplest form, the same laws also hold good in relation to any other system of coordinates $K^{\prime}$ moving in uniform translation relatively to $K . "$
2) Invariance of the speed of light en vacuo (Einstein) [1]: “... light is always propagated in empty space with a definite velocity $c$ which is independent of the state of motion of the emitting body."

If the relative motion of two systems of coordinates $K$, and $K^{\prime}$ is uniform translational, we will call these sets of coordinates: inertial frames of reference. If we are concerning ourselves with only inertial frames of reference; then the definite velocity of light en vacuo $c$, be measured by such observers to be equivalent, is called: universality of the speed of light. 
As we continue we will use observers $O$ and $O^{\prime}$, and system of coordinates $K$ and $K^{\prime}$ interchangeably.

Consider now a photon of light traveling either positively or negatively along the $x$-axis in degenerate complex time. We may define the speed of light

$$
c=\left|\frac{\Delta x}{\Re(\zeta)}\right| .
$$

Eq. (20) holds true for all operational definitions in degenerate complex time. This means universality of the speed of light is completely satisfied in degenerate complex time for all operational definitions and we will show why in $\S 6$. Identical logic can be extended to velocity in general (not only to that of light) and so may be applied in a similar fashion to describe the principle of relativity. We will elaborate in the first fundamental postulate in a later section, but for now let the reader be satisfied with the explanation that the first postulate is independent of any operational definition of time.

At the moment, we will continue as planned by showing the second fundamental postulate is not restricted to degenerate complex time and holds true for two arbitrary observers conditional on operational definition.

\section{$5 \quad$ Space-time intervals in complex time}

The Minkowski space-time has square interval of the form [2]

$$
\Delta s^{2}=-\Delta t^{2}+\Delta x^{2}+\Delta y^{2}+\Delta z^{2}
$$

in coordinates $\left(x^{0}, x^{1}, x^{2}, x^{3}\right)=(t, x, y, z)$, with metric signature $(-,+,+,+)$. By our definition the temporal interval is the magnitude of the complex time vector $\zeta$. Therefore the time interval $\Delta t$ in terms of the real and imaginary 
operators is given

$$
\Delta t=\sqrt{\Re(\zeta)^{2}+\Im(\zeta)^{2}}
$$

We then make such a substitution and have in complex time, space-time interval Eq. (21) takes the form

$$
\Delta s^{2}=-\left[\cos ^{2} \omega+\sin ^{2} \omega\right] \Delta t^{2}+\Delta x^{2}+\Delta y^{2}+\Delta z^{2}
$$

\section{Light speed measurement experiment in com- plex time}

For two frames of reference $O$ and $O^{\prime}$ writing square intervals in complex time we have

$$
\Delta s^{2}=-\left[\cos ^{2} \omega+\sin ^{2} \omega\right] \Delta t^{2}+\Delta x^{2}+\Delta y^{2}+\Delta z^{2}
$$

and

$$
\Delta s^{\prime 2}=-\left[\cos ^{2} \omega^{\prime}+\sin ^{2} \omega^{\prime}\right] \Delta t^{\prime 2}+\Delta x^{\prime 2}+\Delta y^{\prime 2}+\Delta z^{\prime 2}
$$

respectively. We will now stipulate these two frames remain in a state of relative rest and have origins which coincide. In a state of relative rest observers $O$, and $O^{\prime}$ will agree on the spatial intervals in their respective space-times. The observers $O$, and $O^{\prime}$ will also agree on the synchronization of their clocks. But this agreement of time intervals only extends so far as the magnitudes of complex time vectors $\zeta$, and $\zeta^{\prime}$. That is, they may have non-identical operational definitions of time. The space-time intervals may then be written

$$
\Delta s^{2}=-\left[\cos ^{2} \omega+\sin ^{2} \omega\right] \Delta t^{2}+\Delta x^{2}+\Delta y^{2}+\Delta z^{2}
$$


and

$$
\Delta s^{\prime 2}=-\left[\cos ^{2} \omega^{\prime}+\sin ^{2} \omega^{\prime}\right] \Delta t^{2}+\Delta x^{2}+\Delta y^{2}+\Delta z^{2} .
$$

Have the observer in frame $O$ measure a light beam traveling along the $x$-axis in his/her space-time. The square interval of this light beam is measured

$$
\Delta s^{2}=-\left[\cos ^{2} \omega+\sin ^{2} \omega\right] \Delta t^{2}+\Delta x^{2}=0
$$

according to $O$. By construction let us use the common configuration of the Minkowski space-time diagram $(x, t)$ and entertain me by imagining our set up. Space-times for frames $O$ and $O^{\prime}$ share a common spatial $x$-axis but have temporal axes rotated by $\Delta \omega$. This means the light-like interval in $O$ has a geometric projection onto the interval

$$
\Delta s^{\prime 2}=-\cos ^{2}(\Delta \omega) \Delta t^{2}+\Delta x^{2} \geq 0
$$

in the space-time of $O^{\prime}$. In degenerate complex time, we find $O^{\prime}$ will measure the interval Eq. (29) to be null and light speed by definition is universal for all inertial observers. Now consider the case of non-degenerate complex time for arbitrary operational definitions; where in general we find $O^{\prime}$ will measure the light beam to be traveling at the same speed and obtain a null square interval provided $O^{\prime}$ includes the imaginary part of $O$ time which is normal to the space-time diagram of $O^{\prime}$. But this is equivalent to $O^{\prime}$ adopting the operational definition of $O$. Eq. (29) becomes

$$
\Delta s^{\prime 2}=-\left[\cos ^{2}(\Delta \omega)+\sin ^{2}(\Delta \omega)\right] \Delta t^{2}+\Delta x^{2}=0
$$

Given the geometric projection described by Eq. (29) is independent of uniform relative velocity between $O$ and $O^{\prime}$; we have shown agreement on light speed for 
inertial frames of reference is conditional on the relative degeneracy of operational definitions. Generalizing the definition of light speed to complex time for two observers with arbitrary operational definitions we find that each observer will measure the other's light beam to travel at

$$
c=\left|\frac{\Delta x}{\Delta t \cos (\Delta \omega)}\right|
$$

using their respective operational definitions of time. We notice in degenerate complex time it happens to be $\cos (\Delta \omega)= \pm 1$ for all operational definitions. It is an immediate consequence of light speed invariance that upon extension of time into the complex plane, inertial frames $O$ and $O^{\prime}$ will not agree on the value of light's speed when operational definitions are relatively non-degenerate. Applying a geometrization of units such that $c=1$ via the usual prescription for some observer $O$, assuming the operational definition of $O$ is real

$$
c=\left|\frac{\Delta x}{\Re(\zeta)}\right|=1
$$

But then for an observer $O^{\prime}$ with arbitrary operational definition in non-degenerate complex time, the speed of the same light beam is measured

$$
c=\left|\frac{\Delta x}{\Re\left(\zeta^{\prime}\right)}\right|=\left|\frac{1}{\cos (\Delta \omega)}\right| \geq 1 .
$$

In the spirit of postulation, let the relativity of real and imaginary temporal components in complex time be raised to the status of postulate. Furthermore, let this principle be invoked on the basis we suppose there exists no preferred direction in complex time. 


\section{Generalization of the postulates of special rel- ativity to general complex time}

As mentioned in $\S 4$, the principle of relativity only superficially involves the velocity of observers. That is, invariance of physical laws under uniform translational motion of two coordinate systems $K$, and $K^{\prime}$ is independent of any operational definition of time. In the simplest of terms, whether or not observers agree on relative velocity has no influence on the same physical laws applying for each. As we have shown in the case of light, discrepancy of measurement provided by arbitrary operational definitions of $K$, and $K^{\prime}$ may arise solely as the result of invariant physical laws. For these reasons, extension of time into the complex plane offers no new perspective on the principle of relativity. Except that it may serve as an example of a physical principle which is independent of operational definitions.

However, we have found through the rigor of our definitions upon extending time into the complex plane, universality of the speed of light holds true provided all inertial observers involved have operational definitions of time which are relatively degenerate.

\section{Conclusion}

Universality of the speed of light, it seems, has guided the general opinion that there exists an absolute operational definition of time. And while degenerate complex time provides the conditions necessary for light speed universality by our definition, in general complex time this is the trivial case. Here it was shown that complex time may be non-degenerate and have light speed universality arise from the relative degeneracy of operational definitions. This opens the possibility our Universe may most accurately be described in non-degenerate complex 
time, which is locally relatively degenerate. In the context of complex time as we have defined it, physical theories which adopt light speed universality as a fundamental postulate may be categorized either: (1) Having globally degenerate complex time. (2) Having globally non-degenerate complex time which is locally relatively degenerate.

\section{References}

[1] H. A. Lorentz, A. Einstein, H. Minkowski, and H. Weyl, The Principle of Relativity: A Collection of Original Memoirs on the Special and General Theory of Relativity, edited by A. Sommerfeld, p. 111 and p. 38, (Dover Publications, New York, 1952).

[2] B. F. Schutz, A First Course in General Relativity, p. 9, (Cambridge University Press, New York, 2009). 